\title{
A Novel Psychovisual Threshold on Large DCT for Image Compression
}

\author{
Nur Azman Abu' ${ }^{1}$ and Ferda Ernawan ${ }^{1,2}$ \\ ${ }^{1}$ Faculty of Information and Communication Technology, Universiti Teknikal Malaysia Melaka, 76100 Melaka, Malaysia \\ ${ }^{2}$ Faculty of Computer Science, Universitas Dian Nuswantoro, Semarang 50131, Indonesia \\ Correspondence should be addressed to Ferda Ernawan; ferda1902@gmail.com
}

Received 21 February 2014; Revised 20 August 2014; Accepted 6 October 2014

Academic Editor: Ahmad T. Azar

Copyright (C) 2015 N. A. Abu and F. Ernawan. This is an open access article distributed under the Creative Commons Attribution License, which permits unrestricted use, distribution, and reproduction in any medium, provided the original work is properly cited.

A psychovisual experiment prescribes the quantization values in image compression. The quantization process is used as a threshold of the human visual system tolerance to reduce the amount of encoded transform coefficients. It is very challenging to generate an optimal quantization value based on the contribution of the transform coefficient at each frequency order. The psychovisual threshold represents the sensitivity of the human visual perception at each frequency order to the image reconstruction. An ideal contribution of the transform at each frequency order will be the primitive of the psychovisual threshold in image compression. This research study proposes a psychovisual threshold on the large discrete cosine transform (DCT) image block which will be used to automatically generate the much needed quantization tables. The proposed psychovisual threshold will be used to prescribe the quantization values at each frequency order. The psychovisual threshold on the large image block provides significant improvement in the quality of output images. The experimental results on large quantization tables from psychovisual threshold produce largely free artifacts in the visual output image. Besides, the experimental results show that the concept of psychovisual threshold produces better quality image at the higher compression rate than JPEG image compression.

\section{Introduction}

Most digital cameras implement a popular block transform in image coding [1]. The sequential block-based coding is a popular technique since it is compact and easy to implement. In standard JPEG image compression, an image is compressed by one block of $8 \times 8$ pixels at a time. The block-based DCT coding has prevailed at reducing interpixel statistical redundancy [2]. However, in order to achieve high compression ratio, $8 \times 8$ image block size with default JPEG quantization tables produces discontinuity of intensities among adjacent image blocks. These discontinuities of the intensity image between two adjacent image blocks cause a visual artifact due to interblock correlations [3] in image reconstruction. Block transform coding always results in blocking artifact at low bit rate. Blocking artifact is one of the most annoying problems [4].

The blocking effect becomes visible within smooth regions where adjacent block is highly correlated with an input image. Since the $8 \times 8$ blocks of image pixels are coded separately, the correlation among spatially adjacent blocks provides boundary blocks when the image is reconstructed [5]. The artifact images in the compressed output are introduced by two factors. First, the transform coefficients coming out of the quantization process are rounded and then inadequately dequantized. Second, the blocking artifacts appear by the pixel intensity value discontinuities which occur along block boundaries [6]. These blocking artifacts are often visually observable.

This research pays a serious attention to the role of block size in transform image coding. Referring to JPEG image compression, the block of $8 \times 8$ image pixels based line coding has provided a low computational complexity. Previously, a compression scheme on $16 \times 16$ block has been investigated by Pennebaker and Mitchell [7] for image compression. This scheme does not provide an improvement on the image compression due to lack of progress on the central processing 
unit in terms of its computing power at the time. The larger $16 \times 16$ block requires an extra image buffering and a higher precision in internal calculations. Nowadays, the technology of the central processing unit grows rapidly in terms of its computing power. Therefore, two-dimensional image transform on larger blocks is now practically efficient to operate on image compression.

In the previous research, the psychovisual threshold has been investigated on $8 \times 8$ image block size [ $8-15$ ]. This paper proposes psychovisual threshold on the large image block of $256 \times 256$ DCT in order to reduce significant blocking effect within the boundary of small image block. This paper also discusses the process and apparatus to generate $256 \times 256$ quantization tables via a psychovisual threshold.

The organization of this paper is as follows. The next section provides a brief overview on a psychoacoustic model. Section 3 discusses a brief description of the $256 \times 256$ discrete cosine transform. Section 4 explains the development of psychovisual threshold on the large discrete cosine transform in image compression. Section 5 discusses a quality measurement on compressed output images. Section 6 shows the experimental results of $256 \times 256$ quantization tables from psychovisual threshold in image compression. Lastly, Section 7 concludes this paper.

\section{The Principle of Psychoacoustic Model}

Psychoacoustics is the study on how humans perceive sound or human hearing. Psychoacoustic studies show that the sound can only be heard at certain or higher sound pressure levels (SPL) across frequency order [16]. The psychoacoustics indicates that human hearing sensation has a selective sensitivity to different frequencies [17]. In the noise-free environment, the human ear audibility requires different loudness across various frequency orders. The sound loudness that the human audibility can hear is called the absolute hearing threshold [18] as depicted in Figure 1.

The principle of the psychoacoustic model by incrementing the sound pressure level one bark at a time has been used to detect audibility of human hearing threshold. The same principle of the psychoacoustic technique has been used to measure the psychovisual threshold in image compression by incrementing image frequency signal one-unit scale at a time. A block of image signals as represented by a transform coefficient at a given frequency is incremented one at a time on each frequency order. A psychovisual threshold has been conducted for image compression by calculating the just noticeable difference (JND) of the compressed image from the original image. This research will investigate the contribution of the transformed coefficient on each frequency order to the compressed output image. The average reconstruction error from the contribution of the DCT coefficients in image reconstruction will be the primitive of psychovisual threshold in image compression. This quantitative experiment has been conducted on $256 \times 256$ image blocks.

\section{Discrete Cosine Transform}

The two-dimensional discrete cosine transform [19] has been widely used in image processing applications. The DCT is

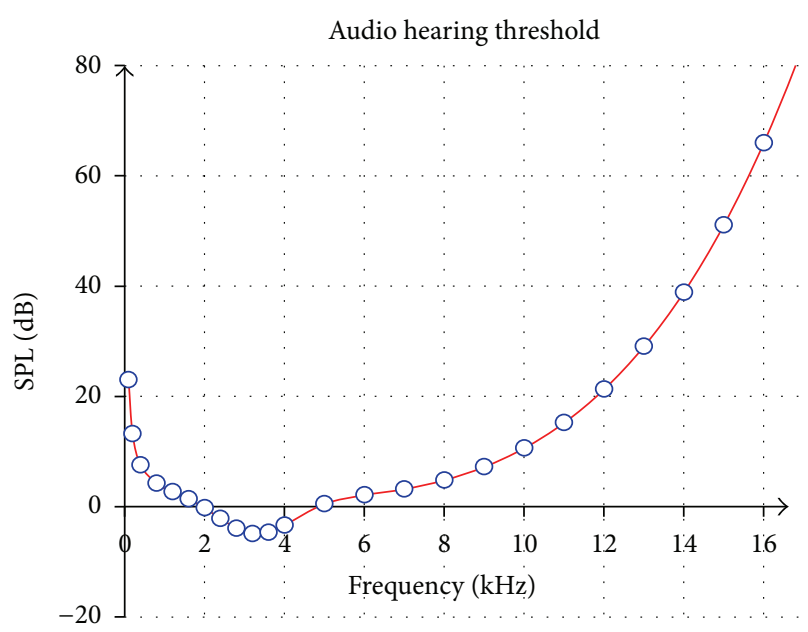

FIgURE 1: The absolute threshold of hearing under quiet condition.

used to transform the pixel values to the spatial frequencies. These spatial frequencies represent the detailed level of image information. The standard JPEG compression uses $8 \times 8 \mathrm{DCT}$ as shown in Figure 2 in image compression.

This paper proposes a large image block of $N \times N$ DCT set $C_{n}(x)$ of size $N=256$ which can be generated iteratively as follows:

$$
\begin{aligned}
& C_{0}(x)=\frac{1}{\sqrt{N}}, \\
& C_{1}(x)=\sqrt{\frac{2}{N}} \cos \frac{(2 x+1) 1 \pi}{2 N}, \\
& C_{2}(x)=\sqrt{\frac{2}{N}} \cos \frac{(2 x+1) 2 \pi}{2 N}, \\
& C_{3}(x)=\sqrt{\frac{2}{N}} \cos \frac{(2 x+1) 3 \pi}{2 N},
\end{aligned}
$$

for $x=0,1,2, \ldots, N-1$. The first four one-dimensional $256 \times$ 256 DCT above are depicted in Figure 3 for visual purposes.

The kernel for the DCT is derived from the following definition [20]:

$$
g=\lambda(u) \cos \frac{(2 x+1) u \pi}{2 N}
$$

where

$$
\lambda(u)= \begin{cases}\frac{1}{\sqrt{N}}, & u=0 \\ \sqrt{\frac{2}{N}}, & u>0,\end{cases}
$$




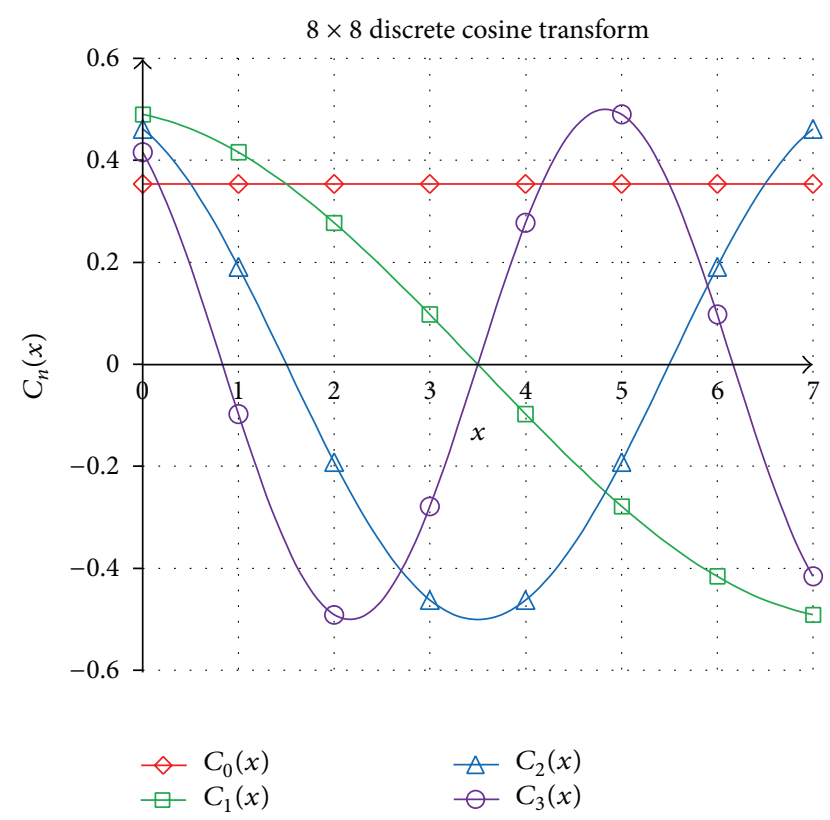

FIgURE 2: The first four $8 \times 8$ DCT of $\operatorname{set} C_{n}(x)$ for $n=0,1,2,3$.

for $x=0,1,2, \ldots, N-1$ and $u=0,1,2, \ldots, N-1$. The definition of the two-dimensional DCT of an input image $A$ and output image $B$ is given as follows [19]:

$$
B_{p q}=\alpha_{p} \beta_{q} \sum_{m=0}^{M-1} \sum_{n=0}^{N-1} A_{m n} \cos \frac{\pi(2 m+1) p}{2 M} \cos \frac{\pi(2 n+1) q}{2 N}
$$

for $p=0,1,2, \ldots, M-1$ and $q=0,1,2, \ldots, N-1$ where

$$
\alpha_{p}=\left\{\begin{array}{ll}
\frac{1}{\sqrt{M}}, & p=0 \\
\sqrt{\frac{2}{M}}, & p>0
\end{array}, \quad \beta_{q}= \begin{cases}\frac{1}{\sqrt{N}}, & q=0 \\
\sqrt{\frac{2}{N}}, & q>0\end{cases}\right.
$$

The inverse of two-dimensional DCT is given as follows:

$$
A_{p q}=\sum_{m=0}^{M-1} \sum_{n=0}^{N-1} \alpha_{p} \beta_{q} B_{m n} \cos \frac{\pi(2 m+1) p}{2 M} \cos \frac{\pi(2 n+1) q}{2 N}
$$

for $p=0,1,2, \ldots, M-1$ and $q=0,1,2, \ldots, N-1$. The image input is subdivided into $M \times N$ blocks of image pixels. The DCT is used to transform each pixel in the $256 \times 256$ image block pixel into the frequency transform domain. The outputs of transforming $256 \times 256$ image blocks of frequency signals are 65536 DCT coefficients. The first coefficient in the upper left corner of the array basis function is called the direct current (DC) coefficient and the rest of the coefficients are called the alternating current (AC) coefficients. DC coefficient provides an average value over the $256 \times 256$ block domain.

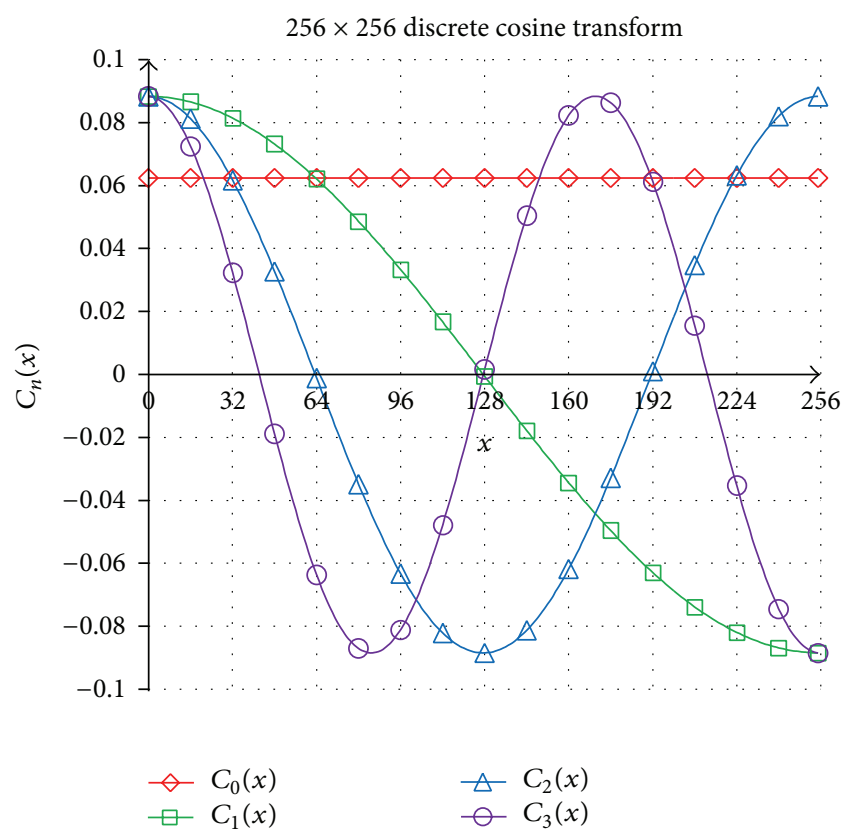

Figure 3: The first four $256 \times 256$ DCT of set $C_{n}(x)$ for $n=0,1,2,3$.

\section{Psychovisual Threshold on Large Discrete Cosine Transform}

In this quantitative experiment, the DCT coefficients on each frequency order are incremented concurrently one at a time from 1 to 255. The impact of incrementing DCT coefficients one at a time is measured by average absolute reconstruction error (ARE). The contribution of DCT coefficients to the quality image reconstruction and compression rate is analyzed on each frequency order. In order to develop psychovisual threshold on $256 \times 256$ DCT, ARE on each frequency order is set as a smooth curve reconstruction error. An ideal average reconstruction error score of an incrementing DCT coefficient on each frequency order on luminance and chrominance for 40 real images is shown in Figure 4.

An ideal finer curve of ARE on a given order from zero to the maximum frequency order 510 for luminance and chrominance channels is presented by the red curve and blue curve, respectively. These finer curves of absolute reconstruction error are set as the psychovisual threshold on $256 \times$ 256 DCT. The contribution of an ideal error reconstruction for each frequency order is determined by two factors, its contribution to the quality on image reconstruction and the bit rates on image compression. The smooth curve of ARE is interpolated by a polynomial that represents the psychovisual error threshold on $256 \times 256$ DCT in image compression. With reference to Figure 4, this paper proposes the new psychovisual thresholds on $256 \times 256$ DCT basis function for luminance $f_{V L}$ and chrominance $f_{V R}$ which are simplified as follows:

$$
\begin{aligned}
f_{V L}(x)= & -0.0000000000001435 x^{5} \\
& +0.000000000011 x^{4}+0.000000046 x^{3} \\
& -0.000009 x^{2}+0.00088 x+0.2352
\end{aligned}
$$




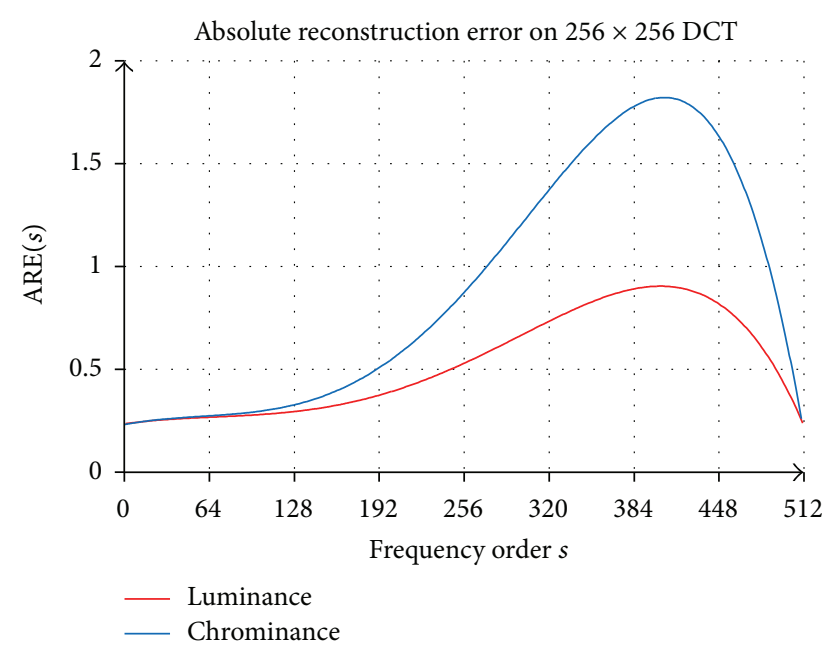

Figure 4: Average absolute reconstruction error of incrementing DCT coefficients on $256 \times 256$ DCT luminance and chrominance for 40 real images.

$$
\begin{aligned}
f_{V R}(x)= & -0.000000000000457 x^{5} \\
& +0.000000000156 x^{4}+0.00000006 x^{3} \\
& -0.0000128 x^{2}+0.0012 x+0.2309
\end{aligned}
$$

for $x=0,1,2, \ldots, 510$, where $x$ is a frequency order on $256 \times 256$ image block. Further, these thresholds are used to generate smoother $256 \times 256$ quantization values for image compression. The $256 \times 256$ quantization table has 511 frequency orders from order 0 until order 510. The order 0 resides in the top left most corner of the quantization matrix index of $Q(0,0)$. The first order represents the quantization value of $Q(1,0)$ and $Q(0,1)$. The second order represents $Q(2,0), Q(1,1)$, and $Q(0,2)$. For each frequency order, the same quantization value is assigned to them.

Due to the large size of these new $256 \times 256$ quantization values, it is not possible to present the whole quantization matrix within a limited space in this paper. Therefore, the $256 \times 256$ quantization values are presented by traversing the quantization table on each frequency order in zigzag pattern as shown in Table 1 . Table 1 presents onedimensional index of quantization table on each frequency order. Each index represents the quantization value at those frequency orders. The new finer quantization tables from psychovisual threshold on $256 \times 256$ DCT for luminance and chrominance are shown in Tables 2 and 3, respectively. The visualization of the whole quantization values on each frequency order from the psychovisual threshold for luminance and chrominance channels is depicted in Figure 5.

These new finer $256 \times 256$ quantization tables have been generated from the psychovisual threshold functions in (7). Each traversing array in zigzag pattern represents the quantization value on each quantization order from order 0 to order 510 . This new smoother $256 \times 256$ quantization

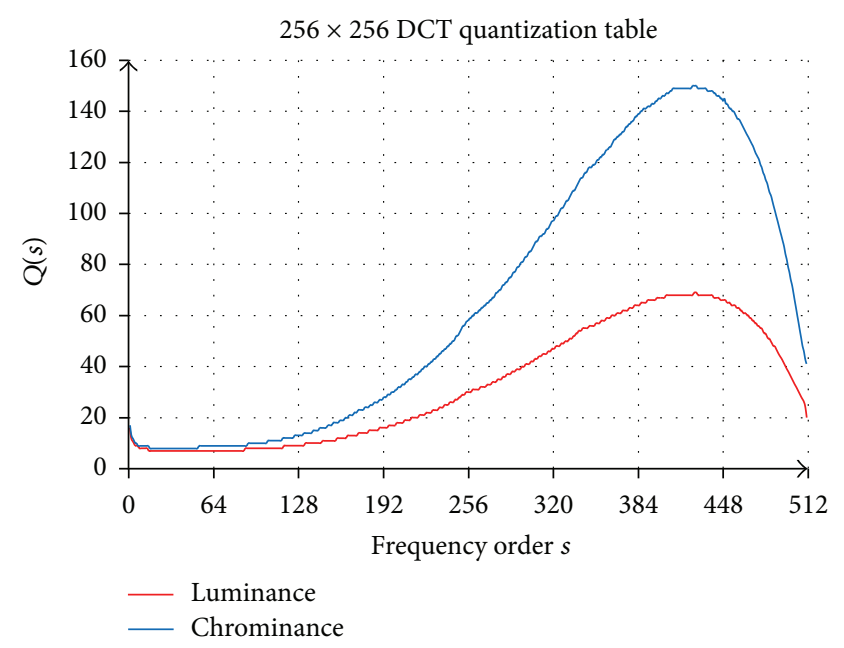

FIGURE 5: The $256 \times 256$ DCT quantization table for luminance and chrominance for image compression.

table for luminance is designed to take smaller value than a quantization table for chrominance. Any slight changes on respective frequency order in the luminance channel will generate significantly greater reconstruction error than a change in chrominance channel. The slight changes by the image intensity on the luminance channel will provide visible textures that can be perceived by human visual systems.

The contribution of the frequency signals to the reconstruction error is mainly concentrated in the low frequency order. Referring to Figure 1, the SPL values on frequency from $2 \mathrm{kHz}$ to $5 \mathrm{kHz}$ are significantly lower. These quantization tables follow the same pattern in order to capture the concept of the psychoacoustic absolute hearing threshold. The quantization values from the psychovisual threshold on chrominance channel are designed to be larger than the quantization values on luminance channel. The human visual system is less sensitive to the chrominance channels as they provide significantly irrelevant image information. The smoother quantization tables will be tested and verified in image compression.

\section{Quality Measurement}

Two statistical evaluations have been conducted in this research project to verify the performances of psychovisual threshold in image compression. In order to gain significantly better performance, the image compression algorithm needs to achieve a trade-off between average bit rates and quality image reconstruction. The conventional quality assessments are employed in this paper. They are average absolute reconstruction error (ARE), means square error (MSE), and peak signal to noise ratio (PSNR). The average reconstruction error can be defined as follows:

$$
\operatorname{ARE}(s)=\frac{1}{\operatorname{MNR}} \sum_{i=0}^{M-1} \sum_{j=0}^{N-1} \sum_{k=0}^{R-1}|g(i, j, k)-f(i, j, k)|,
$$


TABLE 1: The index of quantization value on each frequency order.

\begin{tabular}{|c|c|c|c|c|c|c|c|c|c|c|c|c|c|c|c|c|c|c|c|c|c|c|}
\hline 0 & 1 & 5 & 6 & 14 & 15 & 27 & 28 & 44 & 45 & 65 & 66 & 90 & 91 & 119 & 120 & 152 & 153 & 189 & 190 & 230 & 231 & 275 \\
\hline 2 & 4 & 7 & 13 & & & 29 & 43 & 4 & & 67 & 89 & 92 & 18 & 21 & 51 & & 38 & 191 & 29 & 32 & 274 & 276 \\
\hline 3 & 8 & 12 & 17 & & 30 & 42 & 47 & 6 & & 88 & 93 & 117 & 122 & 150 & 55 & 87 & 92 & 228 & 33 & 273 & 277 & 18 \\
\hline 9 & 11 & 18 & 24 & 31 & 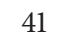 & 48 & 62 & 6 & 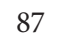 & & 116 & & 149 & 156 & 86 & 93 & 27 & 234 & 272 & 278 & 17 & 19 \\
\hline 10 & 1 & 2. & 3. & 40 & 4 & 61 & 7 & 8 & 95 & 11 & 1 & & 1 & 1 & & 6 & & 71 & 79 & 6 & & 57 \\
\hline 20 & 2 & 3 & 39 & 5 & 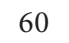 & 71 & 8 & 96 & & & & & 18 & & & & & 30 & 5 & 1 & & 58 \\
\hline 21 & 3 & 38 & 51 & 59 & 72 & 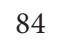 & 9 & & & & 1 & 1 & $1 s$ & 4 & 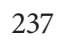 & 207 & & 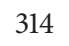 & 2 & 5 & 9 & 92 \\
\hline 35 & 3 & 5 & 58 & & & 98 & & & & & & & 223 & & & & & & 334 & 0 & & 93 \\
\hline 36 & 53 & 5 & 74 & & & & & & & & 19 & & 239 & 201 & & & & J & $\pi 1$ & 0 & 4 & 123 \\
\hline & 56 & 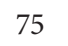 & 81 & & & & & & & & & & 26 & & & & & & 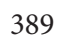 & 395 & 422 & 424 \\
\hline & 7 & 80 & & & & & & & 2 & & & & 28 & 3 & & & & 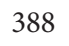 & 6 & 21 & & 450 \\
\hline 77 & 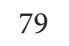 & 102 & 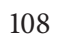 & & & & & & & & & & 30 & & & & & & 20 & 426 & & 51 \\
\hline 78 & 1 & 107 & 1 & & 165 & . & 202 & 218 & 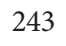 & 26 & 287 & 308 & 328 & 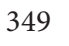 & 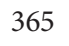 & 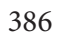 & 398 & 419 & 427 & 448 & 452 & 473 \\
\hline 104 & 106 & 133 & 139 & 166 & 17 & 20 & 21 & 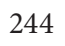 & 26 & 28 & 30 & 329 & 348 & 366 & 385 & 39 & (1) & 428 & 447 & 453 & 472 & 474 \\
\hline 105 & 34 & 138 & 167 & 17 & 204 & 216 & 245 & 26 & 289 & 306 & 330 & 347 & 367 & 384 & 400 & 417 & 429 & 446 & 454 & 471 & 475 & 492 \\
\hline 135 & 137 & 168 & 174 & 205 & 215 & 246 & 260 & 290 & 305 & 33 & 346 & 368 & 383 & 401 & 416 & 430 & 445 & 455 & 470 & 476 & 491 & 493 \\
\hline 136 & 169 & 173 & 206 & 214 & 247 & 259 & 291 & 304 & 332 & 345 & 369 & 382 & 402 & 415 & 431 & 444 & 456 & 469 & 477 & 490 & 494 & 507 \\
\hline 170 & 172 & 207 & 213 & 248 & 258 & 292 & 303 & & 344 & 370 & 381 & 403 & 414 & 432 & 443 & 457 & 468 & 478 & 489 & 495 & 506 & 508 \\
\hline 171 & 208 & 212 & 249 & 2 & 293 & & & & & 380 & 404 & & 433 & 442 & & & 479 & 188 & 496 & 505 & 9 & \\
\hline 209 & 2 & 250 & 256 & & 30 & & & & 379 & 405 & 41 & 434 & 441 & 459 & 466 & 480 & 487 & 497 & 504 & 510 & & \\
\hline 210 & 251 & 255 & 295 & 30 & 336 & & & 37 & 406 & 411 & 435 & 440 & 460 & 465 & 481 & 486 & 498 & 503 & & & & \\
\hline 252 & 254 & & & & 340 & & & & & 436 & 439 & 461 & 464 & 482 & 485 & 499 & 502 & & & & & \\
\hline 253 & 297 & 298 & 338 & 339 & 375 & 376 & 408 & 409 & 437 & 438 & 462 & 463 & 483 & 484 & 500 & 501 & & & & & & \\
\hline
\end{tabular}

TABLE 2: The quantization value on each frequency order for luminance.

\begin{tabular}{|c|c|c|c|c|c|c|c|c|c|c|c|c|c|c|c|c|c|c|c|c|c|c|}
\hline 16 & 12 & 9 & 9 & 7 & 7 & 7 & 7 & 7 & 7 & 7 & 7 & 8 & 8 & 9 & 9 & 11 & 11 & 16 & 16 & 23 & 23 & 34 \\
\hline 11 & 9 & 8 & 8 & 7 & 7 & 7 & 7 & 7 & 7 & 7 & 8 & 8 & 9 & 9 & 11 & 11 & 16 & 16 & 23 & 23 & 34 & 34 \\
\hline 10 & 8 & 8 & 7 & 7 & 7 & 7 & 7 & 7 & 7 & 8 & 8 & 9 & 9 & 11 & 11 & 15 & 16 & 23 & 24 & 34 & 35 & 47 \\
\hline 8 & 8 & 7 & 7 & 7 & 7 & 7 & 7 & 7 & 8 & 8 & 9 & 9 & 11 & 12 & 15 & 16 & 22 & 24 & 33 & 35 & 46 & 47 \\
\hline 8 & 7 & 7 & 7 & 7 & 7 & 7 & 7 & 7 & 8 & 8 & 9 & 11 & 12 & 15 & 16 & 22 & 24 & 33 & 35 & 46 & 47 & 58 \\
\hline 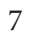 & 7 & 7 & 7 & 7 & 7 & 7 & 7 & 8 & 8 & 9 & 11 & 12 & 15 & 17 & 22 & 24 & 33 & 35 & 46 & 48 & 58 & 58 \\
\hline 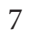 & 7 & 7 & 7 & 7 & 7 & 7 & 8 & 8 & 9 & 11 & 12 & 15 & 17 & 22 & 25 & 33 & 36 & 45 & 48 & 58 & 59 & 66 \\
\hline 7 & 7 & 7 & 7 & 7 & 7 & 8 & 8 & 9 & 11 & 12 & 15 & 17 & 22 & 25 & 32 & 36 & 45 & 48 & 57 & 59 & 66 & 66 \\
\hline 7 & 7 & 7 & 7 & 7 & 8 & 8 & 9 & 10 & 12 & 15 & 17 & 21 & 25 & 32 & 36 & 45 & 48 & 57 & 59 & 66 & 66 & 68 \\
\hline 7 & 7 & 7 & 7 & 8 & 8 & 9 & 10 & 12 & 15 & 17 & 21 & 25 & 32 & 37 & 45 & 49 & 57 & 59 & 65 & 66 & 68 & 68 \\
\hline 7 & 7 & 7 & 8 & 8 & 9 & 10 & 12 & 14 & 17 & 21 & 26 & 32 & 37 & 44 & 49 & 57 & 60 & 65 & 66 & 68 & 69 & 65 \\
\hline 7 & 7 & 8 & 8 & 9 & 10 & 13 & 14 & 18 & 21 & 26 & 32 & 37 & 44 & 49 & 56 & 60 & 65 & 67 & 68 & 69 & 66 & 65 \\
\hline 7 & 8 & 8 & 10 & 10 & 13 & 14 & 18 & 21 & 26 & 31 & 37 & 44 & 50 & 56 & 60 & 65 & 67 & 68 & 69 & 66 & 65 & 56 \\
\hline 8 & 8 & 10 & 10 & 13 & 14 & 18 & 20 & 26 & 31 & 38 & 43 & 50 & 56 & 60 & 65 & 67 & 68 & 68 & 66 & 64 & 57 & 56 \\
\hline 8 & 10 & 10 & 13 & 14 & 18 & 20 & 27 & 31 & 38 & 43 & 50 & 56 & 61 & 64 & 67 & 68 & 68 & 66 & 64 & 57 & 55 & 42 \\
\hline 10 & 10 & 13 & 14 & 18 & 20 & 27 & 31 & 38 & 43 & 51 & 56 & 61 & 64 & 67 & 68 & 68 & 66 & 64 & 58 & 55 & 43 & 41 \\
\hline 10 & 13 & 14 & 18 & 20 & 27 & 31 & 39 & 42 & 51 & 55 & 61 & 64 & 67 & 68 & 68 & 67 & 64 & 58 & 54 & 44 & 40 & 27 \\
\hline 13 & 14 & 19 & 20 & 28 & 31 & 39 & 42 & 51 & 55 & 61 & 64 & 67 & 68 & 68 & 67 & 63 & 59 & 53 & 45 & 39 & 28 & 26 \\
\hline 13 & 19 & 20 & 28 & 30 & 39 & 42 & 52 & 55 & 62 & 64 & 68 & 68 & 68 & 67 & 63 & 59 & 53 & 46 & 38 & 29 & 24 & \\
\hline 19 & 19 & 29 & 30 & 39 & 41 & 52 & 55 & 62 & 63 & 68 & 68 & 68 & 67 & 63 & 60 & 52 & 47 & 37 & 30 & 20 & & \\
\hline 19 & 29 & 30 & 40 & 41 & 53 & 55 & 62 & 63 & 68 & 68 & 68 & 67 & 62 & 60 & 51 & 48 & 36 & 31 & & & & \\
\hline 29 & 30 & 40 & 41 & 53 & 54 & 62 & 63 & 68 & 68 & 68 & 68 & 62 & 61 & 51 & 48 & 35 & 32 & & & & & \\
\hline 30 & 40 & 41 & 54 & 54 & 62 & 63 & 68 & 68 & 68 & 68 & 61 & 61 & 50 & 49 & 34 & 33 & & & & & & \\
\hline
\end{tabular}


TABLE 3: The quantization value on each frequency order for chrominance.

\begin{tabular}{|c|c|c|c|c|c|c|c|c|c|c|c|c|c|c|c|c|c|c|c|c|c|c|}
\hline 17 & 13 & 10 & 9 & 9 & 8 & 8 & 8 & 8 & 8 & 9 & 9 & 10 & 10 & 12 & 12 & 17 & 18 & 27 & 27 & 43 & 44 & 68 \\
\hline 12 & 10 & 9 & 9 & 8 & 8 & 8 & 8 & 8 & 9 & 9 & 10 & 10 & 12 & 12 & 17 & 18 & 27 & 28 & 43 & 44 & 67 & 69 \\
\hline 11 & 9 & 9 & 8 & 8 & 8 & 8 & 8 & 9 & 9 & 9 & 10 & 12 & 12 & 17 & 18 & 26 & 28 & 42 & 45 & 67 & 69 & 97 \\
\hline 9 & 9 & 8 & 8 & 8 & 8 & 8 & 9 & 9 & 9 & 10 & 12 & 12 & 17 & 18 & 26 & 28 & 42 & 45 & 66 & 70 & 96 & 97 \\
\hline 9 & 8 & 8 & 8 & 8 & 8 & 9 & 9 & 9 & 10 & 12 & 13 & 16 & 18 & 26 & 29 & 41 & 46 & 66 & 70 & 95 & 98 & 123 \\
\hline 8 & 8 & 8 & 8 & 8 & 9 & 9 & 9 & 10 & 11 & 13 & 16 & 19 & 26 & 29 & 41 & 46 & 65 & 71 & 94 & 99 & 123 & 124 \\
\hline 8 & 8 & 8 & 8 & 9 & 9 & 9 & 10 & 11 & 13 & 16 & 19 & 25 & 29 & 40 & 47 & 65 & 72 & 94 & 99 & 122 & 125 & 142 \\
\hline 8 & 8 & 9 & 9 & 9 & 9 & 10 & 11 & 13 & 16 & 19 & 25 & 30 & 40 & 47 & 64 & 72 & 93 & 100 & 122 & 125 & 142 & 143 \\
\hline 8 & 9 & 9 & 9 & 9 & 10 & 11 & 13 & 16 & 19 & 25 & 30 & 40 & 48 & 64 & 73 & 92 & 101 & 121 & 126 & 142 & 143 & 149 \\
\hline 9 & 9 & 9 & 9 & 10 & 11 & 13 & 16 & 20 & 24 & 30 & 39 & 48 & 63 & 73 & 92 & 102 & 121 & 126 & 141 & 143 & 149 & 150 \\
\hline 9 & 9 & 9 & 10 & 11 & 13 & 15 & 20 & 24 & 31 & 39 & 49 & 63 & 74 & 91 & 102 & 120 & 127 & 141 & 144 & 149 & 150 & 143 \\
\hline 9 & 9 & 10 & 11 & 14 & 15 & 20 & 24 & 31 & 38 & 49 & 62 & 75 & 91 & 103 & 119 & 128 & 141 & 144 & 149 & 150 & 143 & 142 \\
\hline 9 & 10 & 11 & 14 & 15 & 21 & 24 & 32 & 38 & 50 & 62 & 75 & 90 & 104 & 119 & 129 & 140 & 145 & 149 & 150 & 145 & 141 & 122 \\
\hline 11 & 11 & 14 & 15 & 21 & 23 & 32 & 37 & 50 & 61 & 76 & 89 & 105 & 118 & 129 & 140 & 145 & 149 & 149 & 144 & 141 & 123 & 121 \\
\hline 11 & 14 & 15 & 21 & 23 & 32 & 37 & 51 & 61 & 77 & 89 & 105 & 118 & 130 & 139 & 145 & 149 & 149 & 145 & 140 & 124 & 119 & 89 \\
\hline 14 & 14 & 21 & 23 & 33 & 37 & 52 & 61 & 77 & 88 & 106 & 118 & 130 & 139 & 146 & 149 & 149 & 145 & 140 & 126 & 118 & 91 & 87 \\
\hline 14 & 22 & 23 & 33 & 36 & 52 & 60 & 78 & 87 & 107 & 117 & 131 & 138 & 146 & 149 & 149 & 146 & 139 & 127 & 116 & 93 & 84 & 48 \\
\hline 22 & 23 & 33 & 36 & 53 & 60 & 79 & 86 & 108 & 116 & 131 & 138 & 146 & 149 & 149 & 146 & 138 & 128 & 115 & 95 & 82 & 51 & 46 \\
\hline 22 & 34 & 35 & 54 & 59 & 79 & 85 & 109 & 116 & 132 & 137 & 147 & 149 & 149 & 146 & 137 & 129 & 113 & 97 & 79 & 54 & 43 & \\
\hline 34 & 35 & 55 & 59 & 80 & 85 & 109 & 115 & 132 & 136 & 147 & 149 & 149 & 147 & 137 & 130 & 112 & 99 & 77 & 57 & 41 & & \\
\hline 35 & 56 & 58 & 81 & 84 & 110 & 114 & 133 & 136 & 147 & 149 & 148 & 147 & 136 & 131 & 110 & 101 & 74 & 60 & & & & \\
\hline 56 & 58 & 81 & 83 & 111 & 114 & 134 & 135 & 148 & 149 & 148 & 148 & 135 & 132 & 108 & 103 & 72 & 63 & & & & & \\
\hline 57 & 82 & 83 & 112 & 113 & 134 & 135 & 148 & 149 & 148 & 148 & 134 & 133 & 107 & 105 & 69 & 66 & & & & & & \\
\hline
\end{tabular}

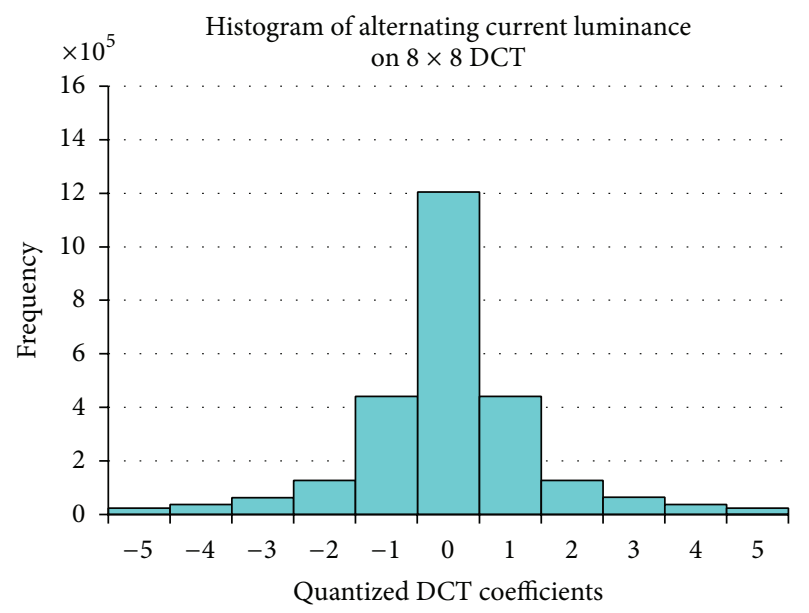

(a)

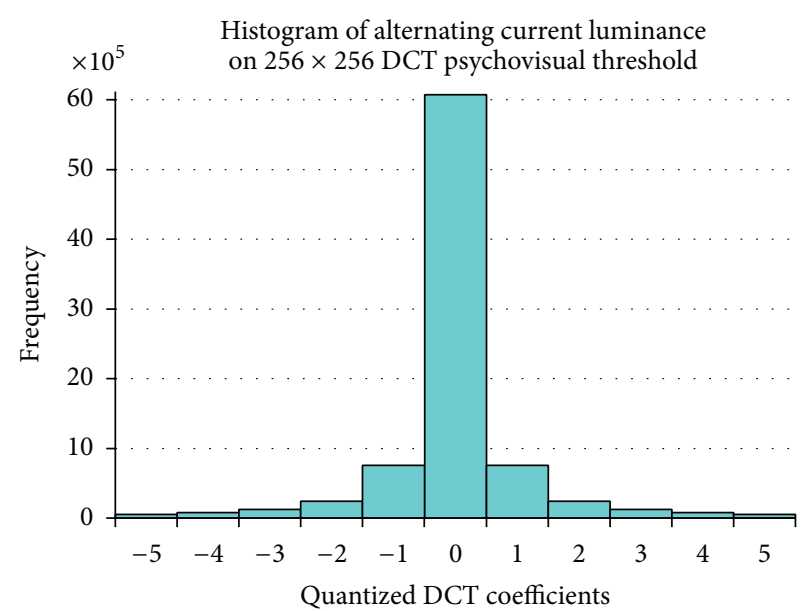

(b)

FIGURE 6: Frequency distribution of the alternating current (AC) coefficients using $8 \times 8$ DCT quantization (a) and $256 \times 256$ DCT quantization from psychovisual threshold (b) on luminance for 40 real images.

where the original image size $M \times N \times R$ refers to the three RGB colors. The MSE calculates the average of the square of the error defined as follows [21]:

$$
\operatorname{MSE}=\sum_{i=0}^{M-1} \sum_{j=0}^{N-1} \sum_{k=0}^{R-1}\|g(i, j, k)-f(i, j, k)\|^{2} .
$$

The standard PSNR is used and calculated to obtain the measure of the quality of the image reconstruction. A higher
PSNR means that the image reconstruction is more similar to the original image [22]. The PSNR is defined as follows:

$$
\operatorname{PSNR}=20 \log _{10}\left(\frac{\operatorname{Max}_{i}}{\sqrt{\mathrm{MSE}}}\right)=10 \log _{10}\left(\frac{255^{2}}{\mathrm{MSE}}\right),
$$

where $\operatorname{Max}_{i}$ is the maximum possible pixel value of the image. Structural similarity index (SSIM), another measurement of image quality, is a method to measure quality by capturing 


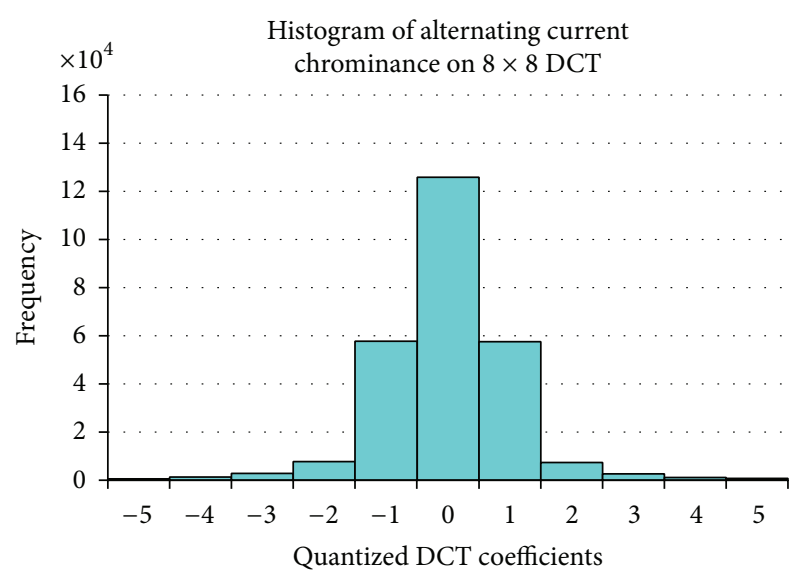

(a)

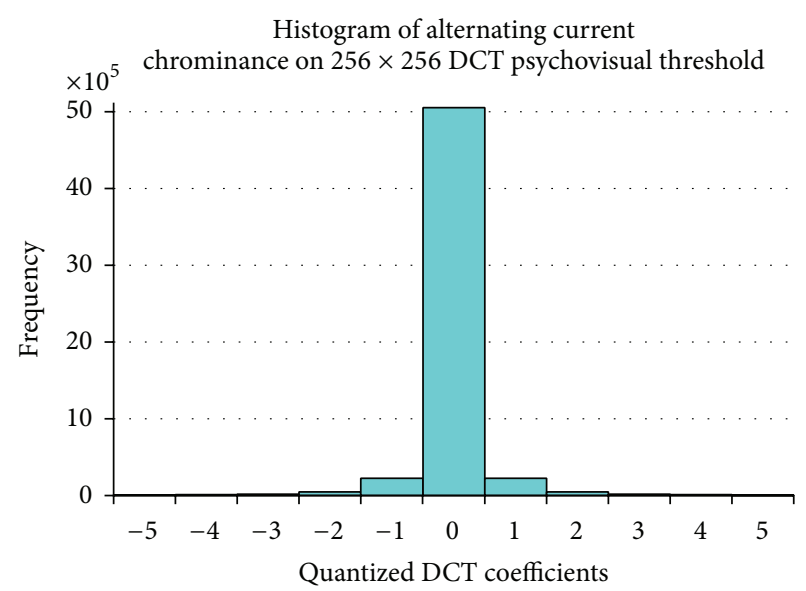

(b)

FIGURE 7: Frequency distribution of the alternating current (AC) coefficients using $8 \times 8$ default JPEG quantization tables (a) and $256 \times 256$ DCT quantization from psychovisual threshold (b) on chrominance for 40 real images.

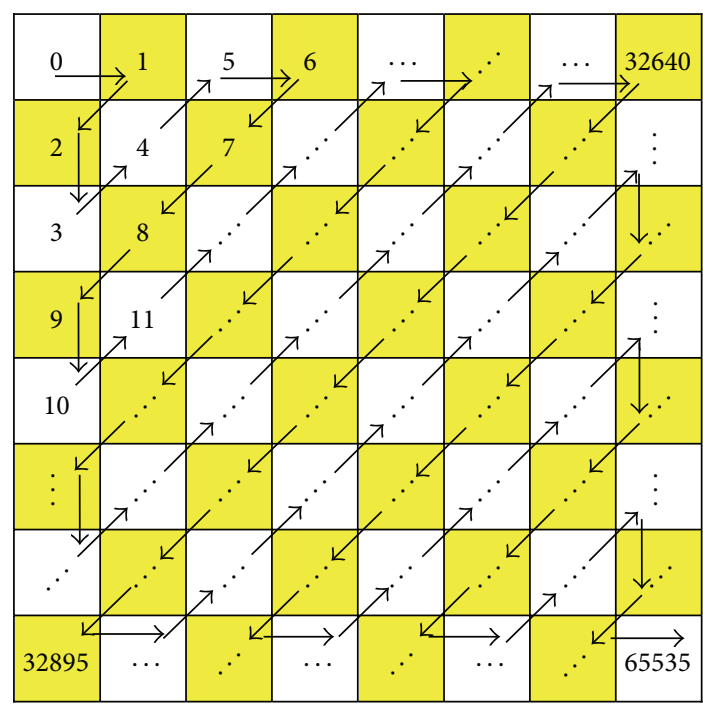

FIGURE 8: Zigzag order of $256 \times 256$ image block.

the similarity between original image and compressed image [23]. The SSIM is defined as follows:

$$
\operatorname{SSIM}(x, y)=[l(x, y)]^{\alpha} \cdot[c(x, y)]^{\beta} \cdot[s(x, y)]^{\gamma},
$$

where $\alpha>0, \beta>0, \gamma>0$ are parameters to adjust the relative importance of the three components. The detailed description is given in [23].

\section{Experimental Results}

This quantitative experiment has been conducted to investigate the performance of a psychovisual threshold on the large image block. The new finer $256 \times 256$ quantization tables have been generated from the psychovisual threshold on $256 \times 256$
DCT. They are tested on 40 real and 40 graphical high fidelity images. An input image consists of $512 \times 512$ colour pixels. The RGB image components are converted to $\mathrm{YCbCr}$ color space. In this experiment, each image is divided into $256 \times 256$ image block pixels; thus each block is transformed into $256 \times 256$ DCT. The DCT coefficients are quantized by new finer $256 \times 256$ quantization tables from psychovisual threshold. The frequency distribution of the transformed coefficients after the quantization process is summarised by histograms in Figures 6 and 7. The histogram of the frequency distribution is obtained after quantization processes. Figures 6 and 7 show a histogram of the frequency distribution after quantization process of $8 \times 8$ default JPEG quantization tables and $256 \times 256$ quantization tables from psychovisual threshold for luminance and chrominance, respectively.

The compression rate focuses mainly on the contribution of the AC coefficients to image compression performance. The frequency coefficients after quantization process consist of many zeros. The frequency distribution of the AC coefficients given by its histogram may predict the compression rate. The higher zeros value on the histogram of frequency distribution means that the image compression output provides lower bit rate to present an image.

According to Figures 6 and 7, the distribution of the frequency coefficients after the quantization process by smoother $256 \times 256$ quantization tables from psychovisual thresholds produces significantly more zeros for both luminance and chrominance channels, respectively. These finer quantization tables produce a smaller standard deviation on AC coefficients from the large $256 \times 256$ image block than the small $8 \times 8$ image block. Hence, it is possible to code large transformed block using smaller number of bits for the same image.

The $256 \times 256$ transform coding consists of $65535 \mathrm{AC}$ coefficients for each regular block. Most AC coefficients are naturally small coming out of quantization process. The 


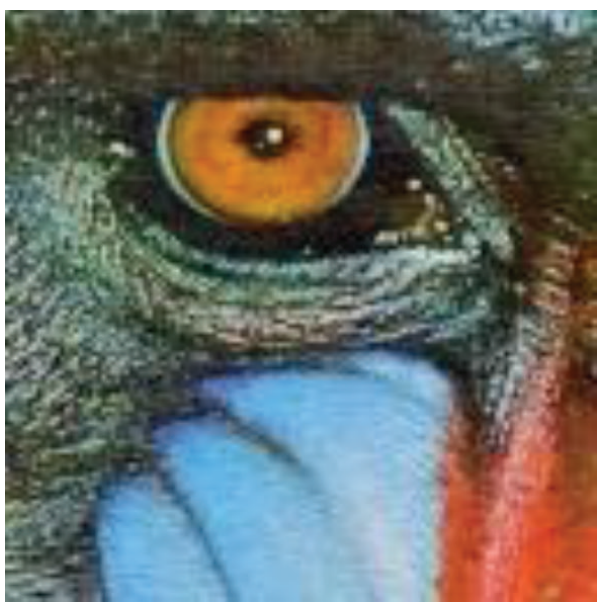

(a)

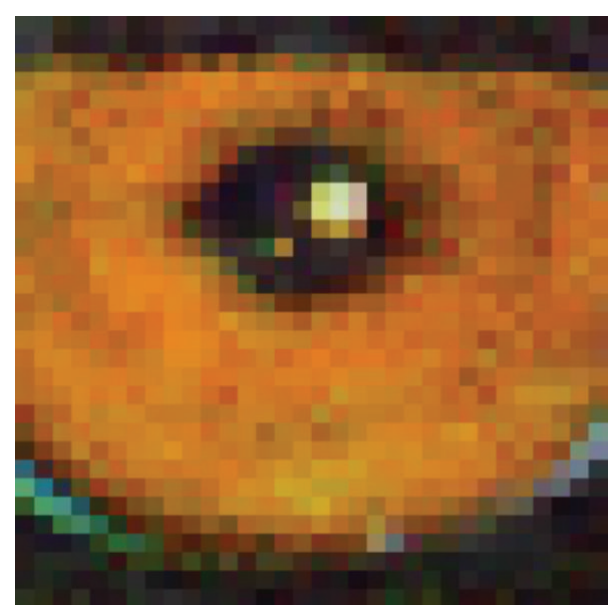

(b)

Figure 9: Original baboon image (a) zoomed in to $400 \%$ (b).

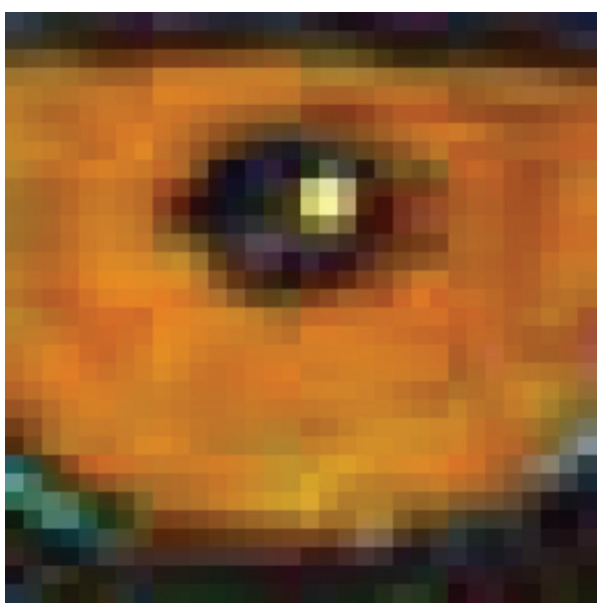

(a)

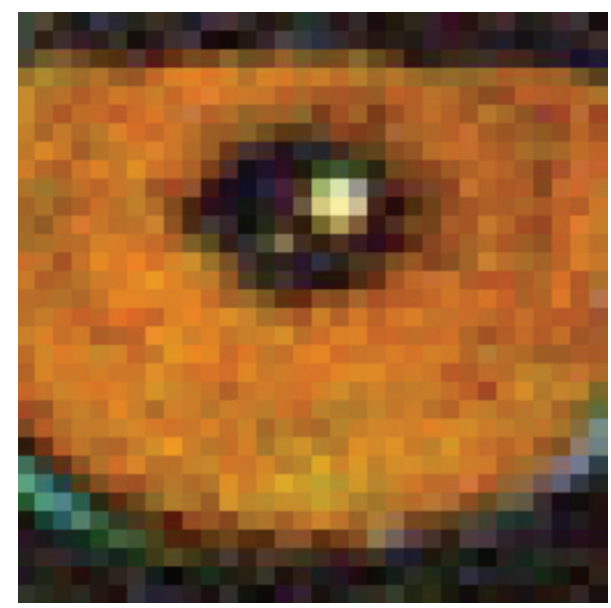

(b)

FIGURE 10: The comparison of visual outputs between $8 \times 8$ JPEG quantization table (a) and $256 \times 256$ quantization tables from psychovisual threshold (b) zoomed in to $400 \%$.

psychovisual threshold on large DCT determines an optimal contribution of the AC coefficients of large transform coding. The new $256 \times 256$ quantization tables from psychovisual threshold are able to reduce down the irrelevant AC coefficients. The distribution of the transform coefficients gives an indication on how much transform coefficients will be encoded by a lossless Huffman coding.

An average Huffman code is calculated from the quantized transform coefficients. After the transformation and quantization of $256 \times 256$ image block are over, the direct current (DC) coefficient is separated from the AC coefficients. The AC coefficients are listed as a traversing array in zigzag pattern as shown in Figure 8.

Next, run length encoding is used to reduce the size of a repeating coefficient in the sequence of the AC coefficients. The coefficient values can be represented compactly by simply indicating the coefficient value and the length of its run wherever it appears. The output of run length coding represents the symbols and the length of occurrence of the symbols. The symbols and variable length of occurrence are used in Huffman coding to retrieve code words and their length of code words. Using these probability values, a set of Huffman code of the symbols can be generated by Huffman tree. Next, the average bit length is calculated to find the average bit length of the AC coefficients.

There are only four DC coefficients under regular $256 \times$ 256 DCT. The maximum code length of the DC coefficient is 16 bits. The DC coefficients are reduced down by 4 bits. The average bit length of the DC coefficients produces 12 bits after the quantization process in image compression. The average bit length of image compression based on default $8 \times 8$ JPEG quantization tables and the finer $256 \times 256$ quantization tables from psychovisual threshold is shown in Table 4 . The experimental results show the new large quantization tables 
TABLE 4: Average bit length of Huffman code of image compression using $8 \times 8$ JPEG compression and $256 \times 256$ JPEG compression using psychovisual threshold for 40 real images and 40 graphical images.

\begin{tabular}{|c|c|c|c|c|}
\hline \multirow{2}{*}{ Average bit length of Huffman code } & \multicolumn{2}{|c|}{$8 \times 8$ JPEG compression } & \multicolumn{2}{|c|}{$256 \times 256$ JPEG compression } \\
\hline & 40 real images & 40 graphic images & 40 real images & 40 graphic images \\
\hline DC luminance & 5.7468 & 5.6722 & 12 & 12 \\
\hline DC chrominance $\mathrm{Cr}$ & 2.7941 & 3.8663 & 12 & 12 \\
\hline $\mathrm{DC}$ chrominance $\mathrm{Cb}$ & 3.1548 & 4.0730 & 12 & 12 \\
\hline AC luminance & 2.8680 & 2.9653 & 2.3031 & 2.6582 \\
\hline AC chrominance $\mathrm{Cr}$ & 2.0951 & 2.5059 & 1.2931 & 2.1450 \\
\hline $\mathrm{AC}$ chrominance $\mathrm{Cb}$ & 2.1845 & 2.5158 & 1.3656 & 2.1855 \\
\hline
\end{tabular}

TABLE 5: The average image reconstruction error using $8 \times 8$ JPEG compression and $256 \times 256$ JPEG compression using psychovisual threshold for 40 real images and 40 graphical images.

\begin{tabular}{|c|c|c|c|c|}
\hline \multirow{2}{*}{ Image measurement } & \multicolumn{2}{|c|}{$8 \times 8$ JPEG compression } & \multicolumn{2}{|c|}{$256 \times 256$ JPEG compression } \\
\hline & 40 real images & 40 graphic images & 40 real images & 40 graphic images \\
\hline ARE & 5.535 & 5.648 & 5.074 & 5.050 \\
\hline MSE & 70.964 & 92.711 & 51.841 & 59.625 \\
\hline PSNR & 31.190 & 31.636 & 32.363 & 33.421 \\
\hline SSIM & 0.956 & 0.957 & 0.961 & 0.960 \\
\hline
\end{tabular}

from psychovisual threshold produce a lower average bit length of Huffman code than the default JPEG quantization tables.

The DCT coefficients from a large image block have been greatly discounted by quantization tables in image compression. An optimal amount of DCT coefficients is investigated by reconstruction error and average bit length of Huffman code. The effect of incrementing DCT coefficient has been explored from this experiment. The average reconstruction error from incrementing DCT coefficients is mainly concentrated in the low frequency order of the image signals.

The new $256 \times 256$ quantization table from the psychovisual threshold produces a lower average bit length of Huffman code in image compression as shown in Table 4 . At the same time, the compressed output images produce a better quality image reconstruction than the regular $8 \times 8$ default JPEG quantization tables as listed in Table 5. The new design on quantization tables from psychovisual threshold performs better by producing higher quality in image reconstruction at lower average bit length of Huffman code.

The average bit size of image compression as presented in Table 6 shows that the finer $256 \times 256$ quantization tables from psychovisual threshold use fewer bits. Therefore, the compression ratio of the difference between a compressed image from the new large quantization table and the original image produces a higher compression ratio than standard JPEG image compression as shown in Table 7. In order to observe the visual quality of the output image, a sample of original baboon right eye is zoomed in to $400 \%$ as depicted on the right of Figure 9.

The image compression output of $256 \times 256$ quantization table from psychovisual threshold is shown on the right of Figure 10. A visual inspection on the output image using $256 \times 256$ quantization tables from psychovisual threshold produces the richer texture on the baboon image. The psychovisual threshold on $256 \times 256$ image block gives an optimal balance between the fidelity on image reconstruction and compression rate. The experimental results show that the psychovisual threshold on large DCT provides minimum image reconstruction error at lower bit rates. The JPEG image compression output as depicted on the left of Figure 10 contains artifact image or blocking effect under regular $8 \times 8$ block transform coding. At the same time, the smoother $256 \times$ 256 quantization tables from psychovisual threshold manage to overcome the blocking effects along the boundary blocks. These finer $256 \times 256$ quantization tables from psychovisual threshold provide high quality image with fewer artifact images. The psychovisual threshold is practically the best measure of an optimal amount of transform coefficients to the image coding.

\section{Conclusion}

This research project has been designed to support large block size in a practical image compression operation in the near future. A step-by-step procedure has been developed to produce psychovisual threshold on $256 \times 256$ block transform. The use of the psychovisual threshold on a large image block is able to overcome the blocking effect or artifact image which often occurs in standard JPEG compression. The psychovisual threshold on discrete transform has been used to determine an optimal amount of frequency transform coefficients to code by generating the much needed quantization tables. Naturally, a frequency transform on large image block is capable of reducing redundancy and better exploiting pixel correlation within the image block. The new set of quantization tables from psychovisual threshold produces 
TABLE 6: The average bit size of $8 \times 8$ JPEG compression and $256 \times 256$ JPEG compression using psychovisual threshold.

$8 \times 8$ JPEG compression

$256 \times 256$ JPEG compression

\begin{tabular}{lccc}
40 real images & 40 graphic images & 40 real images & 40 graphic images \\
\hline $230.997 \mathrm{~Kb}$ & $258.394 \mathrm{~Kb}$ & $158.792 \mathrm{~Kb}$ & $223.652 \mathrm{~Kb}$
\end{tabular}

TABLE 7: The average compression ratio score of $8 \times 8$ JPEG compression and $256 \times 256$ JPEG compression using psychovisual threshold.

$8 \times 8$ JPEG compression

40 real images

3.3247

40 graphic images

2.9722

40 real images

$256 \times 256$ JPEG compression

4.8365

[9] F. Ernawan, N. A. Abu, and N. Suryana, "Adaptive tchebichef moment transform image compression using psychovisual model," Journal of Computer Science, vol. 9, no. 6, pp. 716-725, 2013.

[10] F. Ernawan, N. A. Abu, and N. Suryana, "An adaptive JPEG image compression using psychovisual model," Advanced Science Letters, vol. 20, no. 1, pp. 26-31, 2014.

[11] F. Ernawan, N. A. Abu, and N. Suryana, "An optimal tchebichef moment quantization using psychovisual threshold for image compression," Advanced Science Letters, vol. 20, no. 1, pp. 7074, 2014.

[12] F. Ernawan, N. A. Abu, and N. Suryana, "Integrating a smooth psychovisual threshold into an adaptive JPEG image compression," Journal of Computers, vol. 9, no. 3, pp. 644-653, 2014.

[13] F. Ernawan, N. A. Abu, and N. Suryana, "A psychovisual threshold for generating quantization process in tchebichef moment image compression," Journal of Computers, vol. 9, no. 3, pp. 702-710, 2014.

\section{References}

[1] Y. Wang, X. Mao, and Y. He, "A dual quad-tree based variable block-size coding method," Journal of Visual Communication and Image Representation, vol. 21, no. 8, pp. 889-899, 2010.

[2] L. Ma, D. Zhao, and W. Gao, "Learning-based image restoration for compressed images," Signal Processing: Image Communication, vol. 27, no. 1, pp. 54-65, 2012.

[3] L. Wang, L. Jiao, J. Wu, G. Shi, and Y. Gong, "Lossy-tolossless image compression based on multiplier-less reversible integer time domain lapped transform," Signal Processing: Image Communication, vol. 25, no. 8, pp. 622-632, 2010.

[4] S. Lee and S. J. Park, "A new image quality assessment method to detect and measure strength of blocking artifacts," Signal Processing: Image Communication, vol. 27, no. 1, pp. 31-38, 2012.

[5] J. Singh, S. Singh, D. Singh, and M. Uddin, "A signal adaptive filter for blocking effect reduction of JPEG compressed images," International Journal of Electronics and Communications, vol. 65, no. 10, pp. 827-839, 2011.

[6] M. C. Stamm, S. K. Tjoa, W. S. Lin, and K. J. R. Liu, "Antiforensics of JPEG compression," in Proceedings of the IEEE International Conference on Acoustics, Speech, and Signal Processing (ICASSP '10), pp. 1694-1697, Dallas, Tex, USA, March 2010.

[7] W. B. Pennebaker and J. L. Mitchell, JPEG Still Image Data Compression Standard, Springer, New York, NY, USA, 1993.

[8] F. Ernawan, N. A. Abu, and N. Suryana, "TMT quantization table generation based on psychovisual threshold for image compression," in Proceedings of the International Conference of Information and Communication Technology (ICoICT '13), pp. 202-207, Bandung, Indonesia, March 2013.
[14] N. A. Abu, F. Ernawan, and S. Sahib, "Psychovisual model on discrete orthonormal transform," in Proceedings of the International Conference on Mathematical Sciences and Statistics (ICMSS '13), pp. 309-314, Kuala Lumpur, Malaysia, February 2013.

[15] N. A. Abu, F. Ernawan, and N. Suryana, "A generic psychovisual error threshold for the quantization table generation on JPEG image compression," in Proceedings of the IEEE 9th International Colloquium on Signal Processing and its Applications (CSPA '13), pp. 39-43, Kuala Lumpur, Malaysia, March 2013.

[16] L. Hong-Fu, Z. Cong, and L. Rui-Fan, "Optimization of masking expansion algorithm in psychoacoustic models," in Proceedings of the International Symposium on Intelligence Information Processing and Trusted Computing (IPTC '11), pp. 161-164, Hubei, China, October 2011.

[17] H. Bao and I. M. S. Panahi, "Psychoacoustic active noise control based on delayless subband adaptive filtering," in Proceedings of the IEEE International Conference on Acoustics, Speech, and Signal Processing (ICASSP '10), pp. 341-344, Dallas, Tex, USA, March 2010.

[18] H. Chen and T. L. Yu, "Comparison of psycho acoustic principles and genetic algorithms in audio compression," in Proceedings of the 18th International Conference on Systems Engineering (ICSEng '05), pp. 270-275, Las Vegas, Nev, USA, August 2005.

[19] N. Ahmed, T. Natrajan, and K. R. Rao, "Discrete cosine transform," IEEE Transactions on Computers, vol. C-23, no. 1, pp. 90-93, 1993.

[20] O. Hunt and R. Mukundan, "A comparison of discrete orthogonal basis functions for image compression," in Proceedings of 
the Conference on Image and Vision Computing New Zealand (IVCNZ '04), pp. 53-58, November 2004.

[21] A. Horé and D. Ziou, "Image quality metrics: PSNR vs. SSIM," in Proceedings of the 20th International Conference on Pattern Recognition (ICPR '10), pp. 2366-2369, Istanbul, Turkey, August 2010.

[22] Y.-K. Chen, F.-C. Cheng, and P. Tsai, "A gray-level clustering reduction algorithm with the least PSNR," Expert Systems with Applications, vol. 38, no. 8, pp. 10183-10187, 2011.

[23] C. Yim and A. C. Bovik, "Quality assessment of deblocked images," IEEE Transactions on Image Processing, vol. 20, no. 1, pp. 88-98, 2011. 

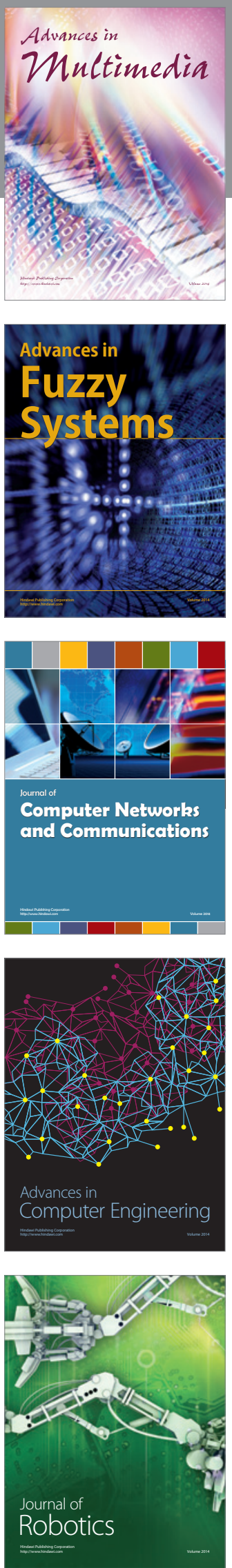

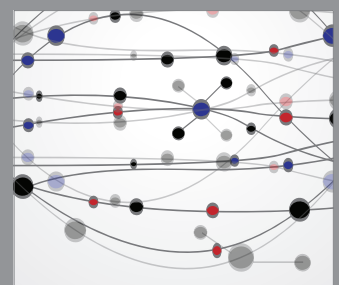

The Scientific World Journal
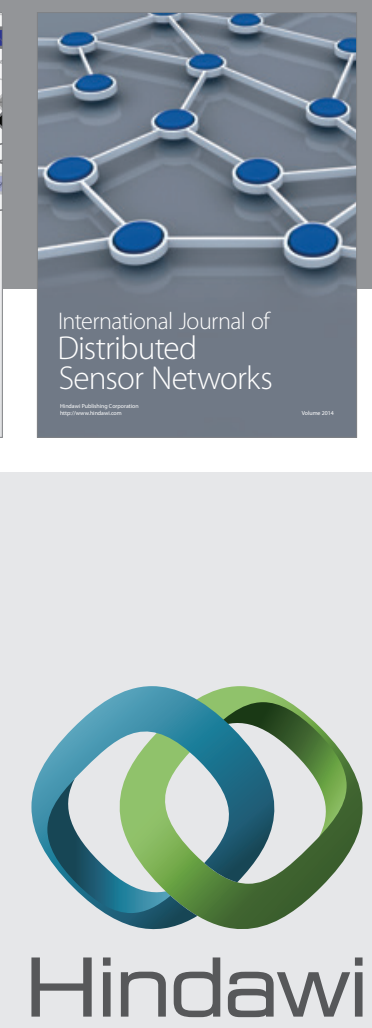

Submit your manuscripts at

http://www.hindawi.com
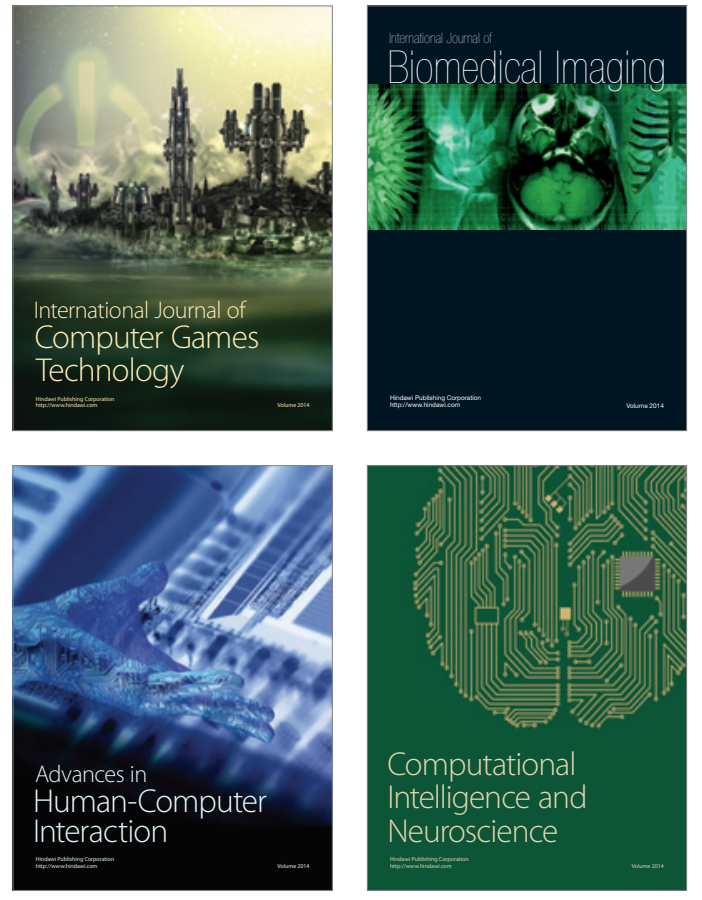
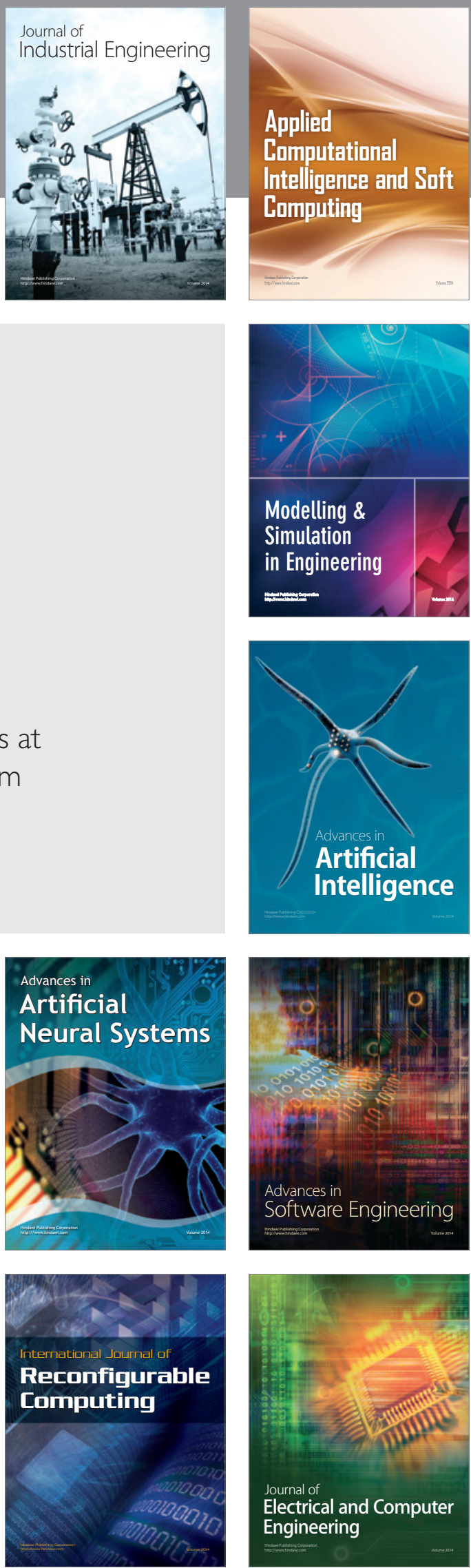